\title{
Fabrication of Micro Solid Metal Needles by Electroplating from PMMA Mold
}

\author{
Y. G. Li ${ }^{1, a^{*}}$, Y. Huang ${ }^{1, b}$, P. Yan ${ }^{1, c}$, S. Sugiyama, ${ }^{2, d}$ \\ ${ }^{1}$ School of Science, Shanghai Institute of Technology, Shanghai, China \\ ${ }^{2}$ Department of Micro Systems, Ritsumeikan University, Kyoto, Japan \\ aygli@sit.edu.cn, ${ }^{b}$ yhuang06@163.com, ${ }^{c}$ pyan91@163.com, dsugiyama@fc.ritsumei.ac.jp \\ * Corresponding Author: ygli@sit.edu.cn
}

\begin{abstract}
Keywords: Moving X-ray lithography, Micro metal needles, Electroplating, Transfer pattern technology
\end{abstract}

Abstract. A nickel solid micro needles array is fabricated by electroplating process based on a PMMA solid micro needles mold. The PMMA mold is fabricated by the moving mask X-ray lithography method. Ni Micro needle array is fabricated by PDMS female mold which is fabricated by using PMMA micro needle array as a master, and transfer PDMS micro needle to Ni micro needle by sputtering the seed layer on the PDMS mold and electroplating metal $\mathrm{Ni}$ to forming $\mathrm{Ni}$ micro needle array. The fabrication method is practical and a Ni micro needle array with the height of 168 $\mu \mathrm{m}$ is obtained. The fabrication technology is practical for the fabrication a complex micro metal structures and it has a high yield.

\section{Introduction}

Three-dimension microfabrication is an important technology in producing various components for micro electro mechanical systems (MEMS). Several process technologies such as LIGA (Lithographie, Galvanoformung, Abformung), DRIE (deep reactive ion etching), excimer laser micromachining have been employed as high aspect ratio micro fabrication techniques [1-7]. The polymer transfer pattern technology for the devices such as micro needle array plays an important role in micro fabrication field and researchers have found its applications in drug delivery system. Because silicon-based micro needle structures tend to be brittle, metallic micro needles are necessary due to its stiffness and toughness. However, few papers on multi-polymer molds transfer technology for fabrication of micro metal needle array are reported. We have reported a method for fabrication PMMA micro needles array with sharp tips by moving X-ray lithography [8]. In this paper, a micro metal needle array fabrication method is described. Firstly, a PMMA male micro needle array mold is fabricated by the moving mask X-ray lithography process; then a soft pattern transfer technique is used to transfer a PMMA male mold to a PDMS female mold. Finally, a nickel micro needle array is fabricated based on the PDMS female mold by the nickel electroplating process. The fabricated micro PMMA needles array mold is 900 micro needles in $1 \mathrm{~cm}^{2}$ chip, the height is $168 \mu \mathrm{m}$ for each micro needle. The fabrication technology has several advantages such as forming micro metal structures with sharp tips, high throughput.

\section{Experime ntal details}

A compact X-ray lithography sources (called AURORA) having the energy of $0.575 \mathrm{GeV}$ and the current of $300 \mathrm{~mA}$ has been operated successfully since 1996 at Ritsumeikan University of Japan. The wavelength range of the X-ray is in the range of $0.15 \mathrm{~nm}$ to $0.73 \mathrm{~nm}$. The four beam lines of the all the 16 beam lines are used for various X-ray lithography sources. Fig. 1 illustrates a scan stage in the exposure chamber for the moving X-ray lithography process. The beam current is about $300 \mathrm{~mA}$ and the peak wavelength is $0.4 \mathrm{~nm}$. The right stage in the Fig. 1 can be moving the resist stage in $\mathrm{x}-\mathrm{y}-\mathrm{Z}$ direction for movement. A beam window size is $38 \mathrm{~mm}$ in length and $5 \mathrm{~mm}$ in width. A X-Y- $\theta$ stage is set up which can move the sample $70 \mathrm{~mm}$ in X direction, $70 \mathrm{~mm}$ in $\mathrm{Z}$ direction to obtain the large area micro structures and rotate freely to exposure by computer control. As shown in Fig.2, an X-ray 
mask for deep X-ray lithography is composed of an X-ray absorber Au on a polyimide film membrane and an Al frame. The thickness of the gold is $3 \mu \mathrm{m}$, the thickness of the polyimide is 38 $\mu \mathrm{m}$ and $\mathrm{Al}$ frame is 5 inch in square.

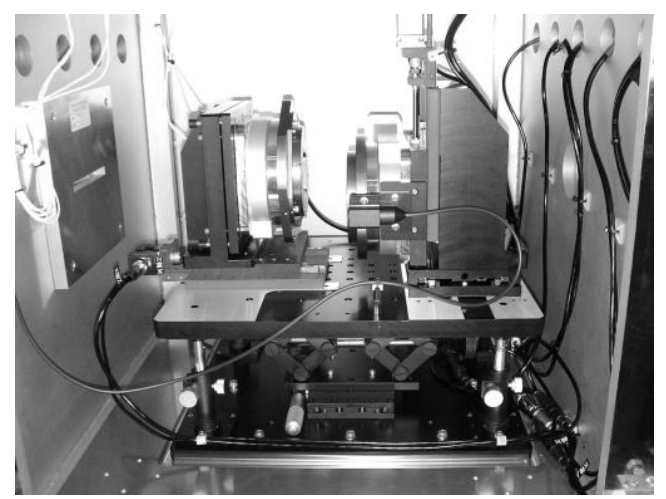

Fig.1 The scan stage for moving X-ray lithography

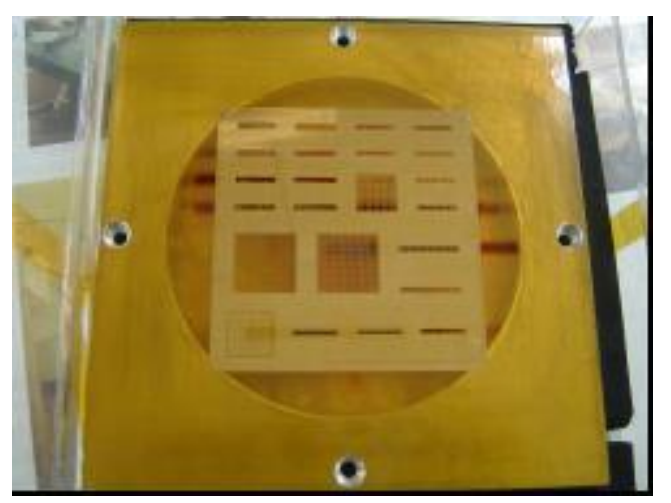

Fig.2 X-ray mask for deep X-ray lithography

Fig. 3 shows the fabrication process for a PMMA male micro needle array mold. An X-ray mask with a group of triangle patterns is used. A PMMA as a positive resist is set up on the resist stage. The mask is moving from upward to downward, the energy distribution of the PMMA sheet is shown in the left of the Fig.3. Due to broken molecule chains and decrease of the molecular weight of PMMA of the exposed region, it is washed away during the development. The unexposed portions of the PMMA are not developed due to unchanged molecular weight. The PMMA micro needles with sharp tips and sloped sidewalls can be fabricated by rotating the mask at 90 degrees and the second X-ray exposure is carried out, as shown in the right of the Fig.3. As a result, the similar cross-section profile of the micro needles with the X-ray mask pattern is fabricated. Scanning X-ray exposure is also employed in order to obtain uniform exposure due to the X-ray Gaussian intensity distribution of X-ray source [9].

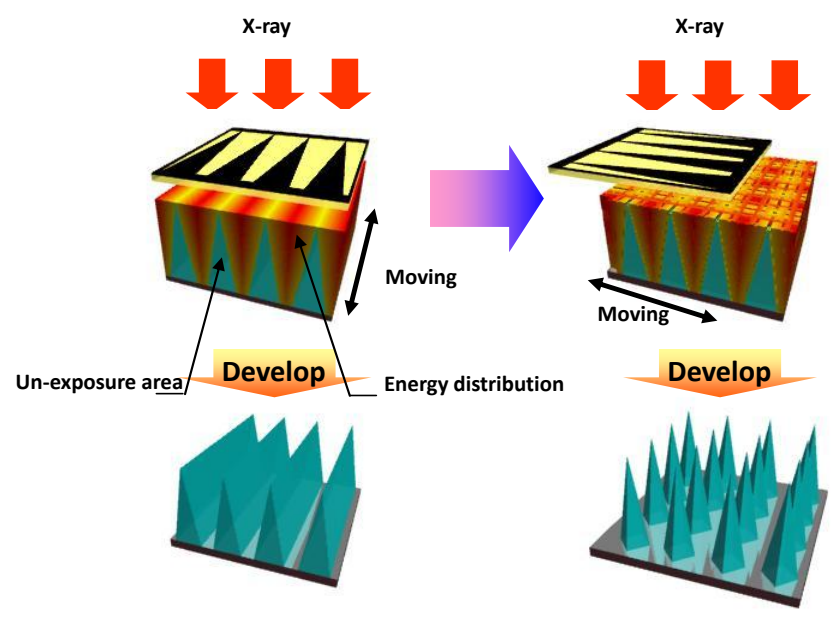

Fig.3 The fabrication process for the PMMA micro needle mold

The PMMA sheet is put carefully in the developer for 150 minutes, then in the stopper again for 15 minutes and rinsed by purified water for another 15 minutes. The solution is stirred at a speed of 200 $\mathrm{r} / \mathrm{min}$ under a constant temperature of $37^{\circ} \mathrm{C}$. The PMMA sheet is left carefully in the developer for 3 hours, then in the stopper again for 15 minutes and rinsed by purified water for another 15 minutes. The solution is stirred at a speed of $200 \mathrm{r} / \mathrm{min}$ under a constant temperature of $37^{\circ} \mathrm{C}$. A fast stirrer speed will destroy the structure and a higher or lower temperature will causes a higher internal stress in the PMMA structure.

In order to fabricate Ni micro needle array, a PDMS female mold is firstly prepared from the fabricated PMMA male mold. PDMS is the most preferred due to its good optical transmittance and 
mold ability. The female PDMS mold having a configuration opposite to that of the PMMA male mold is fabricated by pouring the PDMS solution on the PMMA male mold. A PMMA needle array is transferred to a PDMS female mold because the PDMS is peeled off from the PMMA male mold easily. The fabricated PDMS female mold is about $2 \mathrm{~mm}$ in thickness. A copper seed layer is deposited on the PDMS substrate for formation the conductor layer by depositing a copper film on the substrate under a vacuum. And the thickness of the metal copper $(\mathrm{Cu})$ film may range from $50 \mathrm{~nm}$ to $200 \mathrm{~nm}$. On a clean PDMS plate, a copper layer having a thickness of $200 \mathrm{~nm}$ were deposited by the thermal vapor process under a pressure of about $10^{-6}$ bar. The copper film deposition rate is $0.5 \mathrm{~nm} / \mathrm{s}$. By using PDMS mold with copper seed layer on the PDMS plate, a nickel electroplating is carried out at a low current density. The temperature of a nickel-sultanate solution is $37^{\circ} \mathrm{C}$ and the $\mathrm{pH}$ value of the nickel-sultanate bath is 4.5. The higher or lower of temperature of the solution is affected greatly on the internal stress in the metal needle array. A nickel micro needle having a configuration similar to that of the PMMA mold is fabricated by electroplating process. The nickel micro needles are about $168 \mu \mathrm{m}$ in height.

\section{Fabrication results}

The fabricated PMMA micro male mold was shown in Fig.4. The needle is $200 \mu \mathrm{m}$ in height, the diameter of the top tip is $28 \mu \mathrm{m}$, the diameter of the bottom tip is $78 \mu \mathrm{m}$, and the distance between two needles is $200 \mu \mathrm{m}$. Since the relation between the X-ray dosage and the etching rate has non- linearity, the fabricated needle has decreased in height compare with the designed height and a little deform occurs due to the developing process. Fig.5 shows the fabricated PDMS micro female mold from the PMMA micro male mold. Fig.6 shows fabricated Ni micro needle array from PDMS female mold by the electroplating process. Fig.6 (a) shows the metal micro needle array, Fig.6 (b) shows the single metal micro needle. The height of fabricated nickel micro needles is $168 \mu \mathrm{m}$, the upper diameter of the tip is $13 \mu \mathrm{m}$ and the diameter of the bottom tip is $92 \mu \mathrm{m}$.

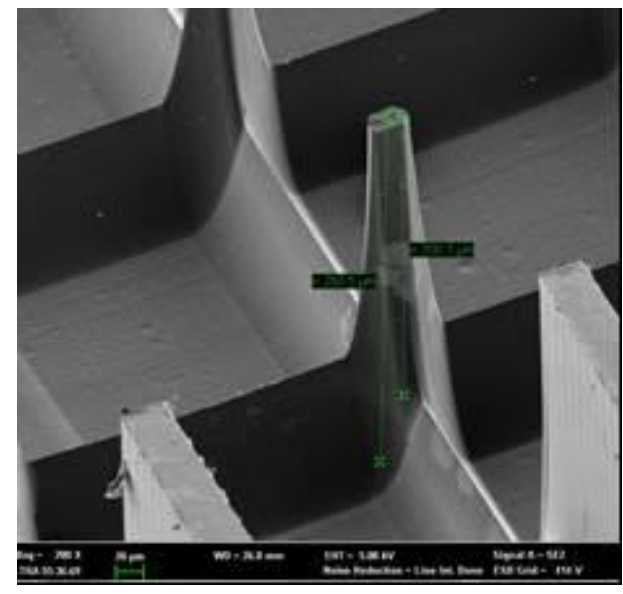

Fig.4 The fabricated PMMA micro male mold

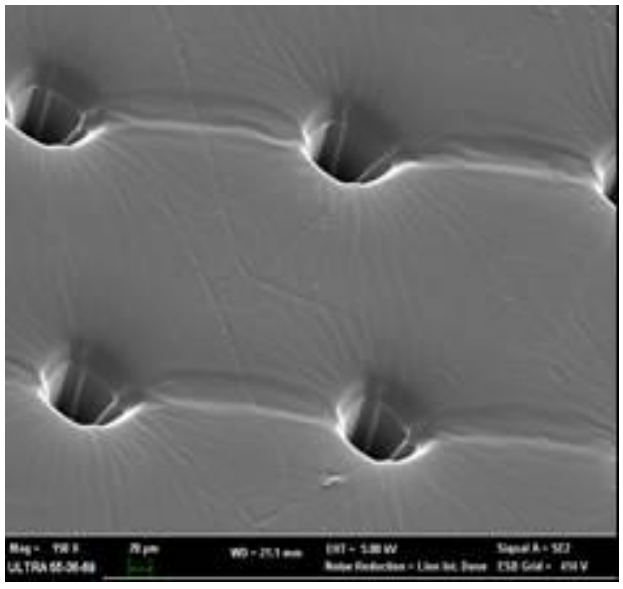

Fig.5 The fabricated PDMS micro female mold 


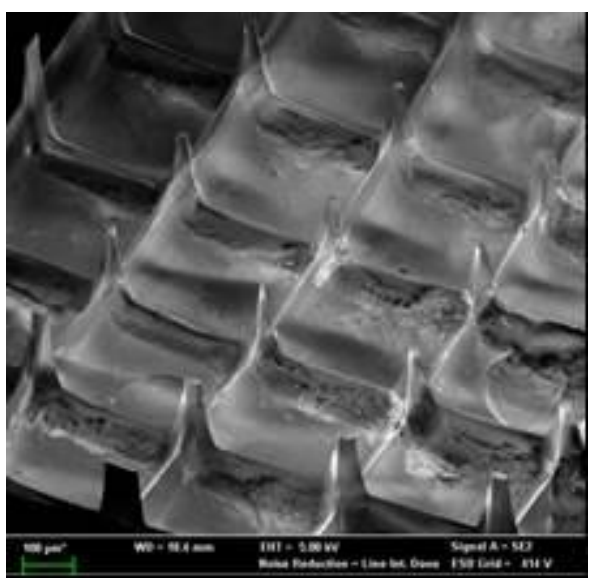

(a)

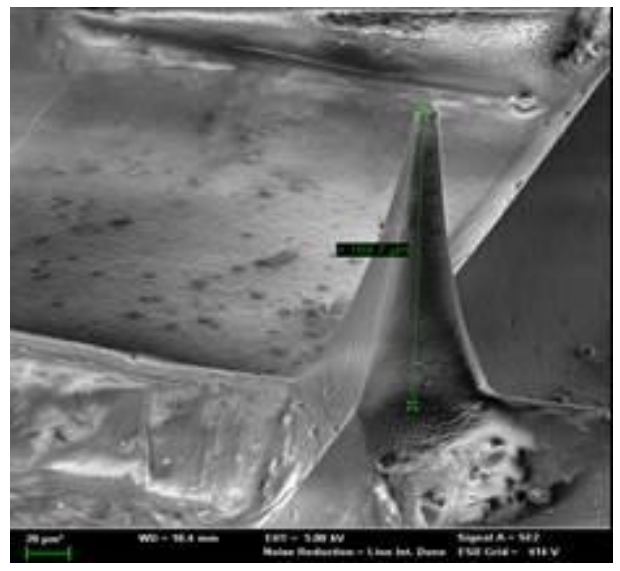

(b)

Fig.6 Fabricated the Ni micro needle, (a) the metal micro needle array, (b) a single metal micro needle

\section{Conclusions}

NiMicro needle array is fabricated by PDMS female mold which is fabricated by using PMMA micro needle array as a master, and transfer PDMS micro needle to Ni micro needle by sputtering the seed layer on the PDMS mold and electroplating metal $\mathrm{Ni}$ to forming $\mathrm{Ni}$ micro needle array. The fabrication method is practical and a Ni micro needle array with the height of $168 \mu \mathrm{m}$ is obtained. PDMS transfer method is a unique method for $3 \mathrm{D}$ metal patterning structures fabrication and creating an almost limitless variety of different features and shapes.

\section{Acknowledgment}

This work was supported by Lian-meng Plan Foundation of Shanghai (LM201441); and Shanghai Institute of Technology Foundation (No.YJ2014-03; No. 10210Q150005).

\section{References}

[1] U. Srinivasan, D. Liepmann, and R. T. Howe: J. Microelectromech. Syst., Vol.10 (2001), p. 17.

[2] L. Muller, A. P. Pisano, and R. T. Howe: J. Microelectromech. Syst., Vol. 10 (2001), p. 550.

[3] S.H Kim,S.H Lee and Y.K Kim: J. Micromech. Microeng. Vol. 12, (2002), p. 128.

[4] C.Ho, W.Hsu : J. Micromech. Microeng. Vol. 14 (2004), p. 356.

[5] M.McNie, D.King and C.Vizard: Microsyst. Technol. Vol. 6 (2000), p. 184.

[6] JL.Tan, J.Tien, DM.Pirone, DS.Gray, K Bhadriraju, and CS. Chen: Proc Natl Acad Sci., Vol.100(2003):,p.1484.

[7] M.Kurosawa and T. Higuchi: Sensors and Acturators A, Vol. 83 (2000), p. 67.

[8] YG. Li, CS. Yang, JQ.Liu and S.Sugiyama: Chin. Phys. Lett. Vol. 28, (2011), p.038101.

[9] YG. Li, S. Sugiyama: Memoirs of the SR Center Ritsumeikan University, Vol. 7 (2005), p. 19. 